\title{
The Los Alamos fission yield evaluation pipeline
}

\author{
M. R. Mumpower ${ }^{1, *}, M$. Verriere ${ }^{1}, A . E$. Lovell ${ }^{1}, T$. Kawano ${ }^{1}, I$. Stetcu $^{1}$, and $P$. Talou $^{2}$ \\ ${ }^{1}$ Theoretical Division, Los Alamos National Laboratory, Los Alamos, NM, 87545, USA \\ ${ }^{2} \mathrm{X}$-Computational Physics Division, Los Alamos National Laboratory, Los Alamos, NM, 87545, USA
}

\begin{abstract}
The nuclear data team at Los Alamos National Laboratory has undertaken a campaign to construct new fission yield evaluation. Significant advances have been made on a number of fronts. Nuclear potential energy surfaces can now be generated with the newly developed MicMac code based off the FiniteRange Liquid-Drop Model (FRLDM). This model can be incorporated into the Los Alamos de-excitation framework codes $\mathrm{BeOH}$ and CGMF to perform modeling of prompt, independent (IFY) and cumulative (CFY) fission yields that take into account prompt and beta-delayed neutrons and photons consistent with decay data. This is in stark contrast to what exists in evaluated nuclear data libraries today, where only a few incident energy points are used with limited physical insights and no consistency between IFY, CFY and decay data. We highlight the latest progress with application of neutron-induced fission of ${ }^{235} \mathrm{U}$ and ${ }^{239} \mathrm{Pu}$.
\end{abstract}

\section{Introduction}

The understanding of fission, or splitting of a heavy nucleus into smaller fragments, is critical for applications of energy production, national security, and fundamental science [1,2]. Los Alamos National Laboratory and partner institutions are currently undertaking a new Fission Product Yield (FPY) evaluation effort for use in such applications. The last major update to the US ENDF FPY evaluation came from the work of Chadwick et al. [3], nearly a decade ago. Since this time, remarkable advancement has been made in theoretical modeling of fission [4-7] and measurements campaigns have produced modern data over a range of excitation energies, see e.g. [8-10]. Our novel evaluation effort seeks to combine these recent advances, focusing on the description of FPY, to independent (IFY) and cumulative (CFY) fission yields that take into account prompt and delayed emission consistent with decay data $[11,12]$. In what follows, we outline a typical workflow of the fission evaluation and report on the progress of theoretical modeling efforts.

\section{The Fission Evaluation Pipeline}

The current Los Alamos fission evaluation effort depends upon the construction of a stateof-the-art platform where many separate codes are combined together under one centralized Python3 framework, NEXUS. The purpose of the NEXUS framework is to handle the inputs and outputs between the various codes in discrete steps. This workflow allows for the parallel

\footnotetext{
*e-mail: mumpower@lanl.gov
} 
development of models, codes and the inclusion of the latest data, providing incremental transactions between relevant steps. The output of a particular step may be tagged with relevant metadata, allowing for the ease of verification and validation. Modifications to data, inputs or outputs and the source code are preserved via Git version control for posterity and reproducibility. The branching and distributed nature of this version control system naturally mimics modern research environments where progress may be made in parallel on many different fronts and then combined at a later time into subsequent parts of an evaluation.

An example of a simple fission evaluation workflow is shown in Fig. 1. Primary fission fragment distributions may be fit to experimental mass yields with an appropriate fitting function. For major actinides, a superposition of five Gaussians has been used in past, and we find this to be suitable for the present work as well. Odd-even staggering in the charge yields can be included using the charge systematics Wahl [13]. In the case no experimental data are available, or when the predictive capabilities of theoretical models meet the required accuracy of evaluation, fragment yields may be generated using the Los Alamos MicMac code, see Sec. 3 for more details. Independent yields, which describe the fission products just after emission of prompt neutrons and $\gamma$-rays, may be constructed given the description of the fission fragment yield and information on the kinetic energy of the fragments. Monte Carlo based codes such as CGMF [14] and FREYA [15] provide access to individual fission events whereas deterministic methods have been employed in the BeOH code [16]. Deterministic methods are of particular importance to evaluation efforts as they probe both low and high statistical regions of the yield curve with equal computational cost. Cumulative yields, those that arise after all $\beta$-decays, and isomeric ratios can be tabulated by soliciting evaluated decay data. We also have another method at our disposal, the input of IFY into the PRISM reaction network code to obtain cumulative yields. We are working towards modifying PRISM to be used to calculate time-dependent applications such as reactor fuel burn up.

Parameter optimization for the various models and codes may happen intermittently over the course of the evaluation process. It is important to keep a detailed record of the particular inputs and outputs for each step in the evaluation. To achieve this in our workflow, we have implemented a special hashing algorithm which uses among other items, a unique universal identifier to track individual calculations. Once the fission yields have been thoroughly tested, official use evaluation files may be generated with the open source DeCE code.

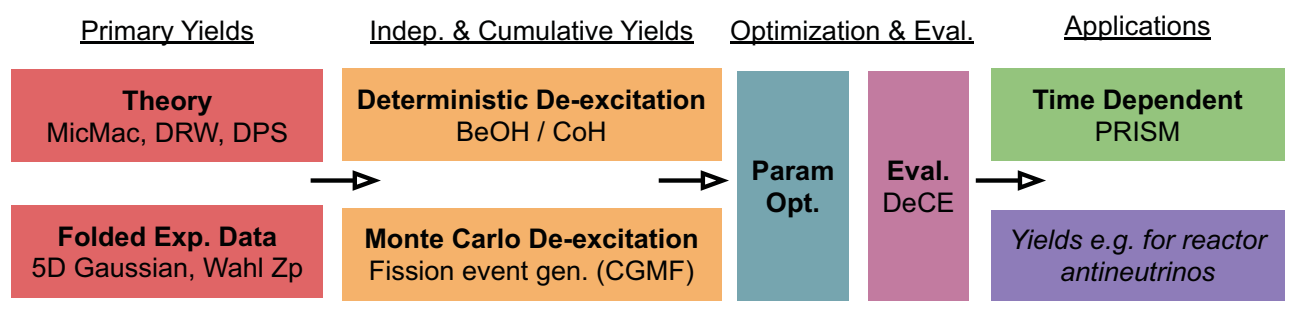

NEXUS

Figure 1. A schematic of some of the codes that comprise the Los Alamos fission evaluation pipeline. The workflow, indicated by arrows, is a combination of many distinct codes woven together by the Python 3 framework, NEXUS. The creation of the evaluation file marks the beginning of public use for a variety of applications. 


\section{Fission Fragment Yields}

Nuclear potential energy surfaces can now be generated with the newly developed MicMac code based off a Matched-Quadratic-Surace shape parameterization of the Finite-Range Liquid-Drop Model (FRLDM), see Dr. Verriere's first-author contribute to this conference proceedings. Several improvements have been made in comparison to older work [17], including an improved Strutinksy implementation and expanded axial harmonic oscillator basis that is extended from 12 to 20 shells. Further advances have been made in the self-consistent calculation of mass and charge yields. These quantities are directly calculated from the wave functions at the point of scission using a particle number projection technique [18]. Using this new method applied to fission fragments allows, for the first time, a prediction of oddeven staggering of charge yields that is seen in data and without invocation of systematics, for example, those of Ref. [13].

The calculation of the fission fragments themselves depends upon the pathways taken through the nuclear potential energy surface as discussed in Refs. [19, 20]. Figure shows the potential energy surfaces for (a) ${ }^{236} \mathrm{U}$ and (b) ${ }^{240} \mathrm{Pu}$ that are used in the calculation of ${ }^{238} \mathrm{U}(\mathrm{n}, \mathrm{f})$ and ${ }^{239} \mathrm{Pu}(\mathrm{n}, \mathrm{f})$. A large ridge (yellow) is seen in both nuclei for small to moderate mass asymmetries, leading to both of these systems fissioning asymmetrically. Using the theoretically calculated nuclear potential, the total kinetic energy of the fragments may also be produced when performing time-dependent calculations, such as with the Langevin approach [21]. Whether the desired accuracy can be achieved for evaluations with such an approach is a subject of current investigation.

(a)

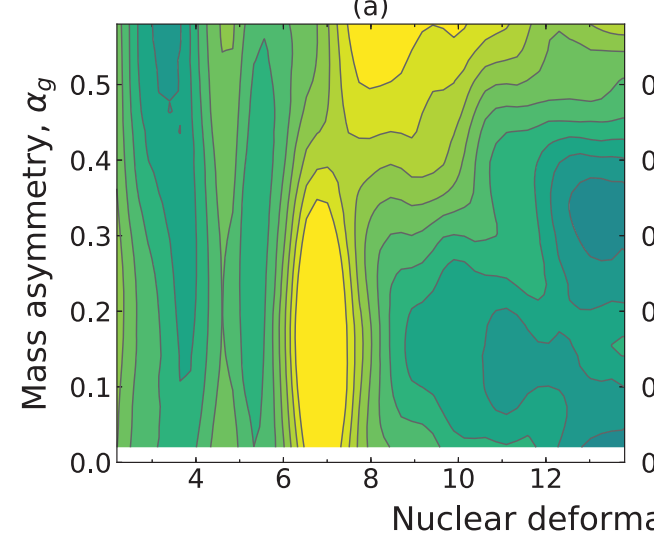

(b)

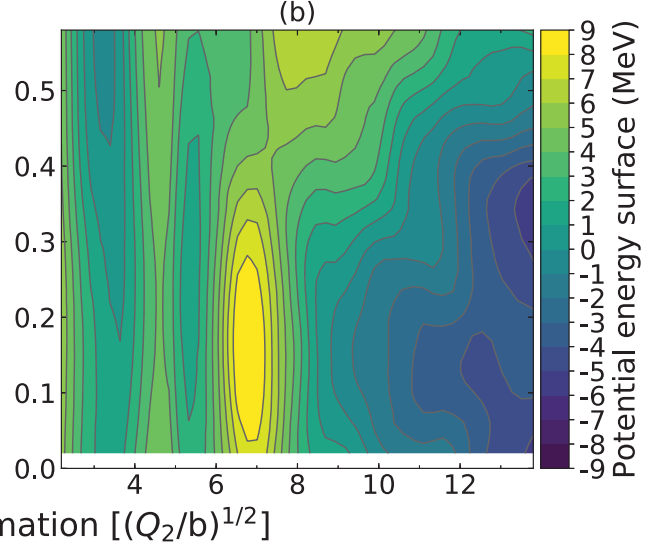

Figure 2. The calculated nuclear potential energy surfaces of (a) ${ }^{236} \mathrm{U}$ and (b) ${ }^{240} \mathrm{Pu}$ using the MicMac code recently developed at Los Alamos. The high-dimensional surfaces are projected down onto mass asymmetry and nuclear deformation. The yellow ridge seen at moderate elongations suggests both systems should fission asymmetrically.

\section{Independent Yields}

Due to the fast timescale of prompt particle emission, independent fission yields are the quantity often measured in experiments. This means that reconstruction of the primary fragments must ensue by invoking a model of the emission. The de-excitation of nascent fragments may be treated theoretically with statistical Hauser-Feshbach calculations [22]. Although there 
have been many studies performed using Monte Carlo methods, such as CGMF [4, 14, 2325], the first attempt at this methodology using the deterministic code $\mathrm{BeOH}$ was recently implemented in Ref. [16] to study reactions with incident neutron energies below secondchance fission. The primary fission fragment yield and information on the total kinetic energy of the fragments are required for such calculations. The average excitation energy in each fragment may be approximated using anisothermal parameter that is defined as the ratio of the effective temperatures of the fragments. From this information, the statistical decay of the fragments may be followed, assuming compound nucleus formation, until all available excitation energy is exhausted.

The primary fragment and independent fission yields for ${ }^{235} \mathrm{U}(\mathrm{n}, \mathrm{f})$ at thermal energies is shown in Fig. 3. The primary mass yield is fit to experimental data using the 5-Gaussian method and the Wahl charge systematics to produce the full yield, $Y(Z, A)$. Total kinetic energy as a function of mass number is also fit to experiment. With this method, the calculated independent fission yields reproduce the trends found in other evaluated libraries such as JENDL.

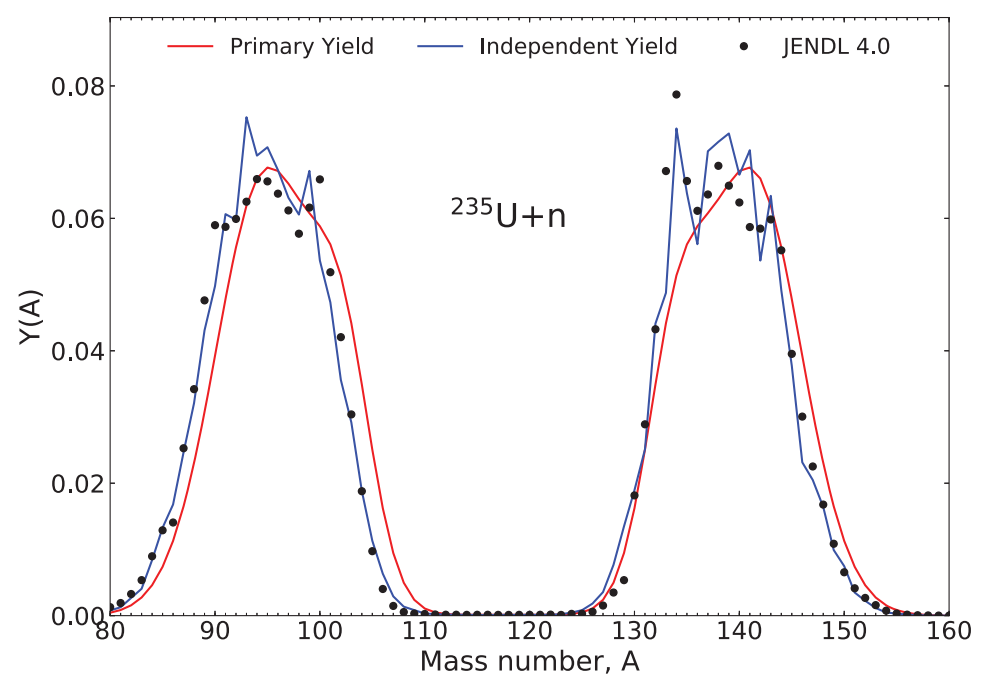

Figure 3. The calculated independent fission yield (blue) after statistical de-excitation of the nascent fragments (red) for the thermal neutron induced fission of ${ }^{235} \mathrm{U}$. Calculations compared to JENDL-4 $[26,27]$.

This procedure also allows the outputting of the properties of emitted prompt neutrons and $\gamma$ rays using well-tested statistical models [28] that are supplemented with evaluated and measured data when applicable [29]. Prompt neutron and $\gamma$-ray spectra associated with ${ }^{235,238} \mathrm{U}(\mathrm{n}, \mathrm{f})$ and ${ }^{239} \mathrm{Pu}(\mathrm{n}, \mathrm{f})$ are currently being studied. Good agreement with the experimental yields and isomeric ratios has been reported recently for the ${ }^{235} \mathrm{U}(\mathrm{n}, \mathrm{f})$ reaction at thermal incident neutron energies [16]. An example of the calculated average number of prompt fission neutrons as a function of the mass of the fragment ca can be seen in Fig. 4 which qualitatively tracks measurements.

While CGMF already takes into account multi-chance fission for incident energies up to $20 \mathrm{MeV}$, work is in progress to extend this formalism beyond the second chance fission threshold using the deterministic method. Additional information is needed for this calculation, in particular the estimation of fission barriers [30]. This is because the transmission 


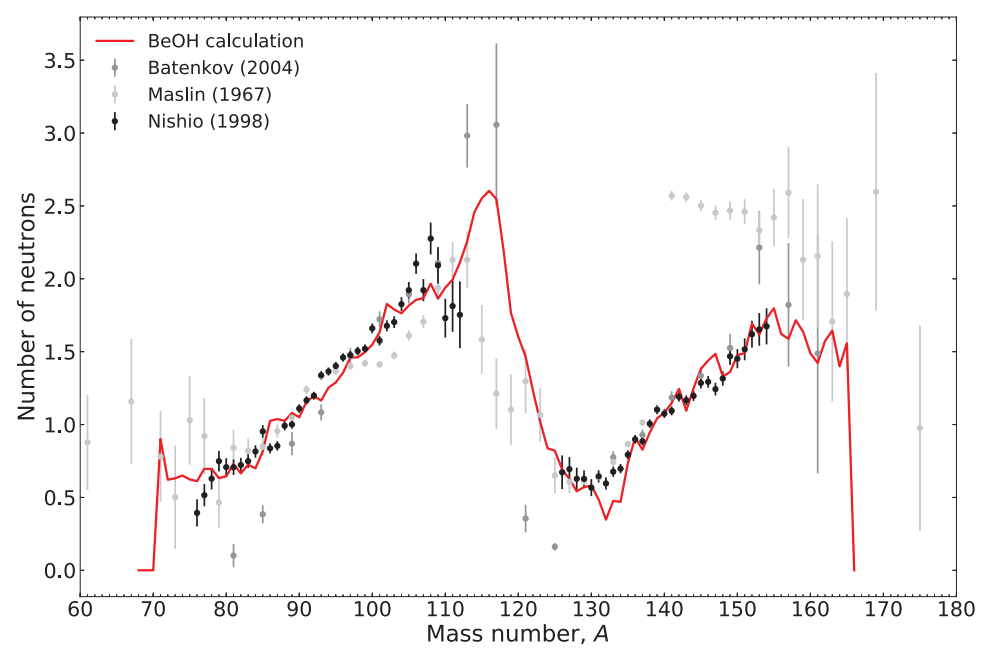

Figure 4. The calculated average number of prompt fission neutrons emitted (red) versus fragment mass number as compared to data (black and gray points) for the thermal neutron induced fission of ${ }^{235} \mathrm{U}$. This calculation corresponds to the independent fission yield (blue line) in Fig. 3.

coefficient for the fission channel is calculated with penetration through a parabolic barrier as in Ref. [31]. The multi-nucleon transfer reaction data of [32] will be a critical test of such modeling capabilities.

\section{Conclusions}

Work on the Los Alamos fission evaluation pipeline has now begun in preparation for the DOE NA-22 program initiative to produce a new evaluation of fission product yields by 2023 . The main goal of this work is to produce a unified framework, that will allow for the consistent evaluation of the fission fragment yields over a range of energies consistent with decay data. Several recent successes on the theoretical side have been reported in this proceedings. The development of the MicMac code for the creation of nuclear potential energy surfaces allows for the calculation of primary fission fragments in both charge and mass. The statistical de-excitation of these nascent fragments may be followed to produce both independent and cumulative yields. Active development on the modeling front is currently underway, with a focus on improving the energy dependence of fission yields.

\section{Acknowledgements}

We thank Mark Chadwick, Ramona Vogt, Jørgen Randrup and Peter Möller for valuable discussions. This work was supported by the US Department of Energy through the Los Alamos National Laboratory. Los Alamos National Laboratory is operated by Triad National Security, LLC, for the National Nuclear Security Administration of U.S. Department of Energy (Contract No. 89233218CNA000001).

\section{References}

[1] R. Capote, D.L. Smith, A. Trkov, EPJ Web of Conferences 8, 04001 (2010) 
[2] P. Talou, R. Vogt, J. Randrup, M.E. Rising, S.A. Pozzi, J. Verbeke, M.T. Andrews, S.D. Clarke, P. Jaffke, M. Jandel et al., The European Physical Journal A 54, 9 (2018)

[3] M. Chadwick, T. Kawano, D. Barr, M.M. Innes, A. Kahler, T. Graves, H. Selby, C. Burns, W. Inkret, A. Keksis et al., Nuclear Data Sheets 111, 2923 (2010), nuclear Reaction Data

[4] P. Talou, B. Becker, T. Kawano, M.B. Chadwick, Y. Danon, Phys. Rev. C 83, 064612 (2011)

[5] R. Vogt, J. Randrup, Nuclear Data Sheets 118, 220 (2014)

[6] T. Kawano, R. Capote, S. Hilaire, P. Chau Huu-Tai, Phys. Rev. C 94, 014612 (2016)

[7] M.R. Mumpower, T. Kawano, P. Möller, Phys. Rev. C94, 064317 (2016), 1608.01956

[8] L. Qi, M. Lebois, J.N. Wilson, A. Chatillon, S. Courtin, G. Fruet, G. Georgiev, D.G. Jenkins, B. Laurent, L. Le Meur et al., Phys. Rev. C 98, 014612 (2018)

[9] N. Fotiades, P. Casoli, P. Jaffke, M. Devlin, R.O. Nelson, T. Granier, P. Talou, T. Ethvignot, Phys. Rev. C99, 024606 (2019)

[10] D. Ramos, M. Caamaño, A. Lemasson, M. Rejmund, L. Audouin, H. Álvarez-Pol, J.D. Frankland, B. Fernández-Domínguez, E. Galiana-Baldó, J. Piot et al., Phys. Rev. Lett. 123, 092503 (2019)

[11] D. Brown, M. Chadwick, R. Capote, A. Kahler, A. Trkov, M. Herman, A. Sonzogni, Y. Danon, A. Carlson, M. Dunn et al., Nuclear Data Sheets 148, 1 (2018), special Issue on Nuclear Reaction Data

[12] G. Audi, F.G. Kondev, M. Wang, W.J. Huang, S. Naimi, Chinese Physics C 41, 030001 (2017)

[13] A.C. Wahl, Los Alamos Report (2002)

[14] B. Becker, P. Talou, T. Kawano, Y. Danon, I. Stetcu, Phys. Rev. C 87, 014617 (2013)

[15] J. Verbeke, J. Randrup, R. Vogt, Computer Physics Communications 222, 263 (2018)

[16] S. Okumura, T. Kawano, P. Jaffke, P. Talou, S. Chiba, Journal of Nuclear Science and Technology 55, 1009 (2018), https://doi.org/10.1080/00223131.2018.1467288

[17] P. Möller, A.J. Sierk, T. Ichikawa, H. Sagawa, Atomic Data and Nuclear Data Tables 109, 1 (2016), 1508.06294

[18] M. Verriere, N. Schunck, T. Kawano, Phys. Rev. C100, 024612 (2019), 1811.05568

[19] J. Randrup, P. Möller, Phys. Rev. Lett. 106, 132503 (2011)

[20] J. Randrup, P. Möller, A.J. Sierk, Phys. Rev. C 84, 034613 (2011)

[21] M.D. Usang, F.A. Ivanyuk, C. Ishizuka, S. Chiba, Phys. Rev. C94, 044602 (2016)

[22] W. Hauser, H. Feshbach, Phys. Rev. 87, 366 (1952)

[23] I. Stetcu, P. Talou, T. Kawano, M. Jandel, Phys. Rev. C 90, 024617 (2014)

[24] P. Jaffke, P. Möller, P. Talou, A.J. Sierk, Phys. Rev. C97, 034608 (2018), 1712 .05511

[25] A.E. Lovell, I. Stetcu, P. Talou, G. Rusev, M. Jandel, Phys. Rev. C 100, 054610 (2019)

[26] K. Shibata, O. Iwamoto, T. Nakagawa, N. Iwamoto, A. Ichihara, S. Kunieda, S. Chiba, K. Furutaka, N. Otuka, T. Ohsawa et al., Journal of Nuclear Science and Technology 48, 1 (2011), https: //doi .org/10.1080/18811248.2011.9711675

[27] K. Shibata, O. Iwamoto, T. Nakagawa, N. Iwamoto, A. Ichihara, S. Kunieda, S. Chiba, J. Katakura, N. Otuka, Journal of Korean Physical Society 59, 1046 (2011)

[28] T. Kawano, S. Chiba, H. Koura, Journal of Nuclear Science and Technology 43, 1 (2006)

[29] M.R. Mumpower, T. Kawano, J.L. Ullmann, M. Krtička, T.M. Sprouse, Phys. Rev. C96, 024612 (2017), 1706.07504 
[30] P. Möller, A.J. Sierk, T. Ichikawa, A. Iwamoto, M. Mumpower, Phys. Rev. C91, 024310 (2015)

[31] D.L. Hill, J.A. Wheeler, Physical Review 89, 1102 (1953)

[32] K. Hirose, K. Nishio, S. Tanaka, R. Léguillon, H. Makii, I. Nishinaka, R. Orlandi, K. Tsukada, J. Smallcombe, M.J. Vermeulen et al., Phys. Rev. Lett. 119, 222501 (2017) 